- 48 denture-bonded crowns evaluated at four years.

- $6 \%$ failure, one crown failing due to fracture, two because of cracks.

- Nil incidence of pulp problems, minimal incidence of debonding.

- Denture bonded crowns may be considered suitable for a variety of incidence on anterior teeth.

\title{
Four year performance of dentine-bonded all-ceramic crowns
}

\author{
F. J. T. Burke ${ }^{1}$
}

\begin{abstract}
Aim This paper reports a prospective evaluation of 59 dentine-bonded crowns placed in a dental school environment for patients, a majority of whom were suffering from tooth substance loss. Results Forty-eight crowns (83\%) were available for examination, with their mean age since placement being 3.9 years. The mean age of the patients in whom the crowns were placed was 37.5 years. Three crowns had failed due to porcelain fractures, an overall failure rate of $6 \%$. Two of the failures were minimal cracks of which the patient was unaware, and one, in an upper premolar tooth, because of crown fracture. No secondary caries was noted, incidence of pulp symptoms or pulp death was nil, and margins were rated as 'excellent' in 42 crowns (86\%). Conclusion The dentine bonded crowns assessed in this study showed excellent retention and low incidence of fracture at four years. This technique would appear to be suitable for a variety of clinical indications, including treatment of tooth substance loss, although the results presented in this study are relatively short term in relation to the anticipated life of restorations.
\end{abstract}

\section{INTRODUCTION}

The dentine-bonded crown has been defined as 'a full coverage restoration in which an all-ceramic crown is bonded to the underlying dentine (and any available enamel) using a resin-composite based luting material, with the bond being mediated by use of a dentine bonding system and a micromechanically retentive ceramic surface.. ${ }^{1}$ Its principal features are therefore:

1. An etchable ceramic - materials such as feldspathic porcelain, aluminous porcelain, and Empress. HF or an $\mathrm{HF} / \mathrm{HCl} / \mathrm{HNO}_{3}$ mixture are generally employed to create the micromechanically retentive intaglio surface.

1Primary Dental Care Research Group, University of Birmingham School of Dentistry, St. Chad's Queensway, Birmingham, B4 6NN

Correspondence to: Professor Trevor Burke

Email:f.j.t.burke@bham.ac.uk

Refereed Paper

Accepted 26 July 2006

DOI: $10.1038 /$ bdj.2007.176

${ }^{\circledR}$ British Dental Journal 2007; 202: 269-273
2. A dentine bonding agent of low film thickness: systems frequently used in the past have been chemically cured, such as Mirage ABC (Chameleon Dental, Kansas City, USA) or Scotchbond Multipurpose Plus (3M ESPE, St. Paul, MN, USA). Light cured materials may be used provided that there is no risk of 'puddles' of material collecting at line angles on polymerisation, as these will be of sufficient thickness to prevent full seating of the crown.

3. A dual cured resin-based luting material, with a sufficient number of shades to allow for slight changes in the shade of the final restoration to be effected by the shade of the luting material: it should, however, be noted that the full polymerisation dual-cure (and chemically cured) resin luting materials may be prevented when low pH self-etching types of dentine bonding agent are employed. ${ }^{2}$
Recently, steps 2 and 3, involving application of etchant, bonding agent and luting material, have been obviated by the introduction of self-adhesive resin-based luting materials, such as RelyX Unicem (3M ESPE, Seefeld, Germany) and Maxcem (Kerr Mfg Co., Orange, CA, USA), making the placement of dentine-bonded restorations significantly less technique sensitive.

The laboratory fracture resistance of dentine bonded crowns has been assessed and considered satisfactory, ${ }^{1,3,4}$ and the aesthetics of dentine-bonded allceramic restorations has been considered to be good, ${ }^{5}$ possibly because of the transmission of light through the restoration because the resin-composite luting materials employed in the technique ${ }^{6}$ are more translucent than conventional acid-base luting materials. Marginal seal has been considered satisfactory. ${ }^{7}$ Minimal preparations have been considered appropriate ${ }^{8,9}$ and it could be considered that the effect upon pulpal tissue should be minimal in view of the reduced tooth 


\begin{tabular}{|c|c|}
\hline Position of labial gingival margin & $\begin{array}{l}\text { Position of labial gingival margin - at, above or below gingival } \\
\text { margin } \\
\text { Position of palatal gingival margin - at, above or below gingival } \\
\text { margin }\end{array}$ \\
\hline Occlusion in intercuspal position & $\begin{array}{l}\text { Assess with articulating paper: } \\
1=\text { Normal } \\
2=\text { Heavy } \\
3=\text { Light }\end{array}$ \\
\hline Gingival health & $\begin{array}{l}\text { To be assessed labially and palatally } \\
0=\text { Optimum gingival health } \\
1=\text { Visible inflammatory changes } \\
2=\text { Bleeding on probing }\end{array}$ \\
\hline Integrity of restoration & $\begin{array}{l}0=\text { Intact } \\
1^{*}=\text { Crack visible on transillumination. (Has crown debonded at } \\
\text { fracture?) } \\
2^{*}=\text { Fracture present. (If fracture present, is it related to } \\
\text { occlusion?) } \\
3^{*}=\text { Crown or inlay lost. (State at which interface debond } \\
\text { occurred.) }\end{array}$ \\
\hline Secondary caries & $\begin{array}{l}\text { Examine all visible margins } \\
0=\text { No visible evidence of caries contiguous with the margin of } \\
\text { the restoration } \\
1^{*}=\text { Caries is evident contiguous with the margin of the } \\
\text { restoration }\end{array}$ \\
\hline Marginal adaptation & $\begin{array}{l}\text { Grade for labial and palatal margins } \\
0=\text { Restoration is contiguous with existing anatomic form, sharp } \\
\text { explorer does not catch } \\
1=\text { Explorer catches, no crevice is visible into which the explorer } \\
\text { will penetrate } \\
2=\text { Crevice at margin, enamel margin exposed } \\
3^{*}=\text { Obvious crevice at margin, dentine or lute exposed }\end{array}$ \\
\hline Marginal discolouration & $\begin{array}{l}\text { Grade for labial and palatal margins } \\
0=\text { No discolouration present } \\
1=\text { Slight staining present, can be polished away. } \\
2=\text { Obvious staining, cannot be polished away } \\
3^{*}=\text { Gross staining }\end{array}$ \\
\hline Colour match & $\begin{array}{l}\text { Examine crown labial margin for colour match to tooth } \\
\text { substance, where visible } \\
0=\text { Very good/good colour match, restoration almost invisible } \\
1=\text { Slight mismatch in colour, shade or translucency } \\
2^{*}=\text { Obvious/gross mismatch, outside the normal range } \\
3^{*}=\text { Gross mismatch }\end{array}$ \\
\hline
\end{tabular}

preparation and the sealing of the dentinal tubules by the dentine bonding system used in the placement technique. In short, the dentine-bonded crown concept has been designed to produce good mechanical properties while producing optimal aesthetics and conserving tooth substance. It may further be considered that the challenge to periodontal tissues should be minimal in view of the potential for well-finished margins, similar to those obtained with porcelain veneers.

The performance of dentine-bonded crowns in previous studies appears promising, with Burke and Qualtrough having reported the retrospective evaluation of 50 dentine bonded crowns, ${ }^{10,11}$ with the results demonstrating continuing success of $91 \%$ of the restorations clinician in a dental school environment for patients, a majority of whom were suffering from tooth substance loss.

\section{MATERIALS AND METHODS}

Ethical approval for the study was obtained from Glasgow Dental Hospi tal Ethical Committee. Patients who were considered to require full-coverage crowns on their anterior teeth were asked if they would consider joining the study. The objectives of the study were explained to patients, who were also given an explanatory letter giving details of the project. Informed consent was given by the patients prior to treatment.

The teeth to be crowned were tested pre-operatively for vitality using an electric pulp tester. Only teeth giving a vital response were included in the study. The teeth were prepared by one operator and the crowns were placed by the same operator. Patients were asked if they were experiencing any sensitivity to hot or cold in the area of the tooth/ teeth to be prepared pre- and post-operatively. Details of any reported sensitivity were recorded.

The tooth preparation included minimal knife-edge preparations at the gingival margin, and reduction of the lingual/palatal surface, as necessary, to provide 1.0-1.5 mm clearance. The crown margins were placed at an equi-gingival level or up to $0.5 \mathrm{~mm}$ supragingivally. Impressions were taken in a vinyl polysiloxane impression material (Express: 3M ESPE, St. Paul, MN, USA) in a stock tray, with opposing arch impressions being taken in alginate. Temporary crowns were provided, constructed in Protemp (ESPE, Seefeld, Germany), and luted with a non-eugenol proprietary cement (Temp Bond Clear: Kerr Mfg. Co, Orange, CA, USA), since a eugenol-containing lute might adversely affect the polymerisation of the resin lute which will be used to place the final crown, ${ }^{13}$ (although the literature on the effect of eugenol on bond strength of adhesive systems is by no means equivocal ${ }^{14}$ ). Crowns were constructed to a standardised technique by one technician in the crown and bridge laboratory in the Glasgow Dental Hospital using feldspathic porcelain on a refractory die. The fitting surface of the finished crowns was etched with proprietary hydrofluoric acid in the laboratory. A Scilane coupling agent (3M ESPE, St. 


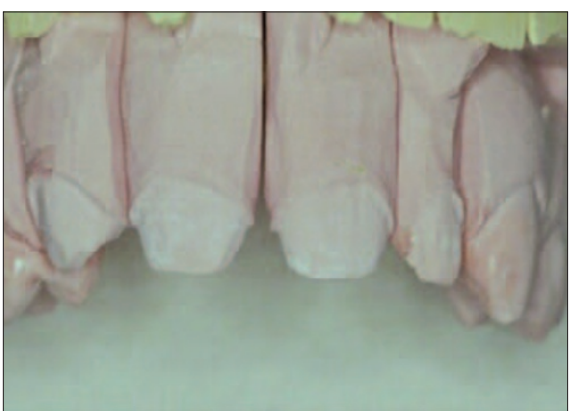

Fig. 1a Minimal preparation associated with dentine-bonded crowns

Paul, MN, USA) was applied in the laboratory and again in the surgery prior to placement.

At the placement appointment the abutment teeth were anaesthetised. The crowns were checked for fit and shade match. (Any crown considered to be of unsatisfactory fit was rejected and a new impression taken.) If necessary, the crown was tried in using the try-in paste, paste B, from the 3M Opal Resin (3M ESPE, St. Paul, MN, USA) luting kit. When a suitable shade of luting material was chosen, the fitting surface of the crown was cleaned with alcohol. If the fit was considered satisfactory, the fitting surface of the crown was cleaned by a 15 second application of 35\% proprietary phosphoric acid, following which the crown was washed with water and dried, and a silane coupling agent applied for one minute. The prepared teeth were isolated using cotton wool rolls and the dentine/enamel prepared in accordance with the manufacturers' instructions for the chemically-curing dentine bonding system Scotchbond Multipurpose Plus (3M ESPE, St. Paul, MN, USA), which also was applied in accordance with the manufacturer's directions. Having chosen the appropriate shade of luting material (which was generally the same as the porcelain shade), the luting material (3M Resin Cement: 3M ESPE, St. Paul, MN, USA) was mixed in accordance with the manufacturer's instructions and applied to the fitting surface of the crown. The crown(s) were placed slowly, with gentle finger pressure. Excess luting material was removed using a probe and felt pads. Floss and Superfloss (Johnson and Johnson, New Jersey, USA) were passed interproximally to remove excess luting material. The luting material was cured by a one minute light cure to the mesial, distal, buccal and palatal/lingual surfaces of the crown using an XL3000

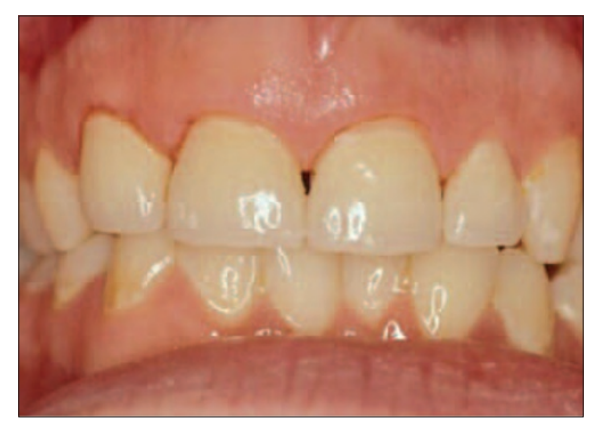

Fig. 1b Dentine-bonded crowns at UR2, UR1, UL1 and UL2 at fit appointment. The crown at UR3 has been 'lateralised'

curing light (3M ESPE St. Paul, MN, USA). (The curing light was operated for 30 seconds prior to use and the light intensity checked before operation.) After polymerisation of the lute, any remaining excess luting material was removed with 15 micron grit fine-tipped, tapered, diamond finishing burs.

The occlusion on the crown was rated as 'heavy', 'normal' or 'light' when assessed using articulating paper.

Patients were reviewed one week after crown placement and, again, annually. Baseline data collected on the first review appointment included periodontal indices, records of mobility and occlusal relationship, the latter being recorded by means of a dual arch tray impression. The patient was asked if s/he had experienced any post-operative sensitivity, to either hot/cold or pressure. Details of any reported sensitivity was recorded. At the recalls, the crowns were assessed according to the criteria described in Table 1, modified from USPHS criteria. ${ }^{15}$ Margins were assessed, overall, as the percentage of crowns for which the margin assessment was codes 0 or 1 .

\section{RESULTS}

Fifty-nine crowns were originally provided for 16 patients. Of these, 47 crowns were placed on maxillary incisor teeth, six on canine teeth, four on mandibular incisor teeth and two on premolar teeth. A majority of the crowns had been placed because of tooth substance loss due to erosive and attritional factors, with 19 of the crowns having been placed in patients whose occlusion was rated as 'heavy', in patients who were considered to have a bruxist habit.

Forty-eight crowns (83\%) were available for examination, with their mean age since placement being 3.9 years (range 3 years to 4.5 years). A selection of these are presented in Figures 1 to 4 .

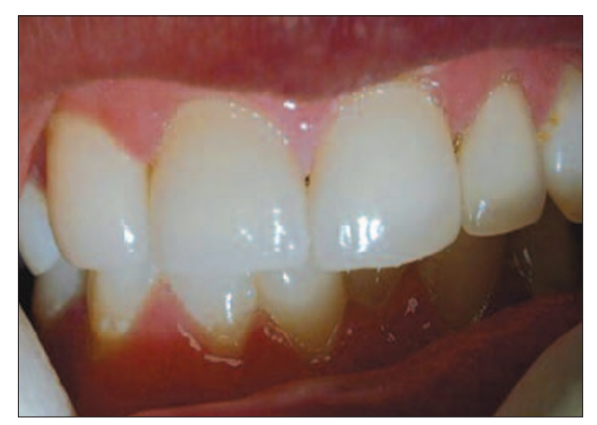

Fig. 1c Crowns in Fig. 1a at 4 years

The mean age of the patients in whom the crowns were placed was 37.5 years (range 22 years to 51 years). One crown had de-bonded at 2.5 years but had been re-cemented, following cleaning and reetching of its fitting surface with HF. This crown was found to be functioning satisfactorily at the time of the fouryear assessment and was therefore not counted as a failure. Three crowns were found to have failed due to porcelain fractures, an overall failure rate of $6 \%$. Two of the failures were minimal cracks of which the patient was unaware, and one, in an upper premolar tooth rated as having a 'heavy' occlusion in a 45-yearold patient, because of crown fracture. No secondary caries was noted, either in the intact or non-intact crowns. Incidence of pulp symptoms or pulp death was nil. Margins were rated as 'excellent' in 42 crowns (86\%). No teeth were found to be mobile.

\section{DISCUSSION}

This paper presents the four-year data on a relatively new restorative technique which has been considered to be of value in the provision of aesthetic all-ceramic crowns in the treatment of teeth affected by tooth substance loss. ${ }^{9}$ The recall rate may be considered satisfactory, and of a similar order to studies of similar design..$^{16,17}$

The results of the present study suggest that catastrophic failure is not a major problem for dentine-bonded crowns placed on anterior teeth, insofar that only one crown failed due to fracture and only one crown debonded. Two crowns had suffered cracks, but the fractured fragments had remained bonded to the underlying tooth substance. These results confirm the results of previous work by Burke and Qualtrough, which indicated a low incidence of fractures and a low (nil) incidence of 


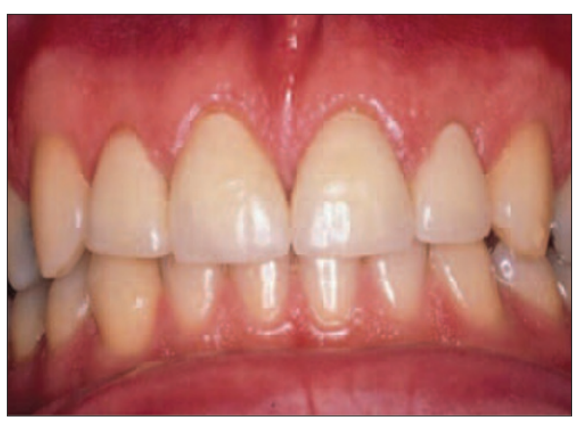

Fig. 2a Dentine-bonded crowns at UR2, UR1, UL1 and UL2 at 4 years

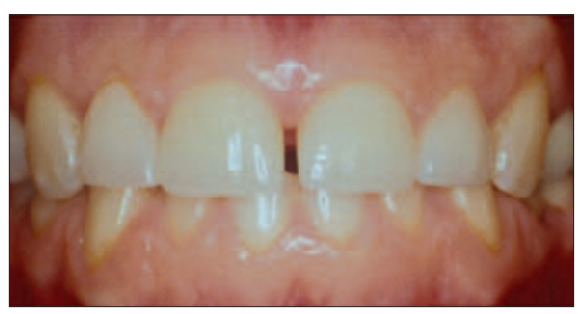

Fig. 3 Dentine-bonded crowns at UR2, UR1 UL1 and UL2 at 4 years. Patient wished to retain her midline diastema

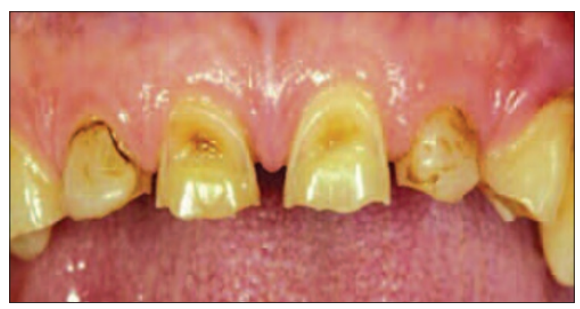

Fig. 4a Tooth substance loss affecting anterior teeth

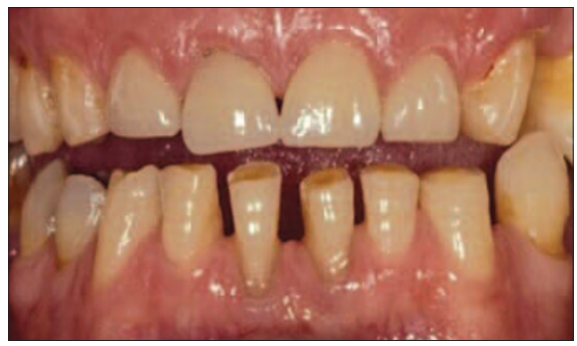

Fig. 4b Dentine-bonded crowns at UR2, UR1, UL1 and UL2 after 4 years. Crown lengthening suggested pre-operatively in order to improve appearance of 'square' teeth but patient declined. He also did not request any treatment for the poor appearance of his lower teeth

crown debonds. ${ }^{10,11}$ They also confirm a report that only three dentine-bonded crowns had cracked but not debonded during 13 years of use of dentine bonded crown techniques in which several thousand crowns were placed (Dr Sverker Toreskog, Personal Communication, Manchester, Nov 1998). Most recently, Etemadi and Smales have reported the five-year performance of resin-bonded porcelain veneer crowns (an alternative name for dentine-bonded crowns), with and without metal reinforcement, finding that $14.5 \%$ of the metal reinforced crowns had failed, compared with 18.6\% of the all-ceramic crowns, a statistically significant difference. ${ }^{12}$ However, the failure rate of $7.8 \%$ in all-ceramic dentine-bonded crowns in anterior teeth is not dissimilar to that reported here. Their study differs from the present work, insofar as the crowns were placed in general dental practice by two specialist practitioners. However, the indications for the placement of dentine-bonded crowns appears to be similar, with the restorations being placed predominantly for aesthetic reasons for damaged teeth and those with defective restorations, while, in the present study, the crowns were generally placed in teeth damaged by tooth wear. In this respect, the criteria generally employed for planning to use dentine bonded crowns in the present study were (a) tooth substance loss palatally and/or labially and, (b) teeth which were suboptimal aesthetically. In cases of tooth substance loss in which there was loss of tooth tissue on the palatal surface but in which the appearance of the labial surface of the tooth was considered satisfactory by the patient, a composite restoration would have been bonded to the worn palatal surface and/ or incisal edge, rather than preparing the tooth for a dentine-bonded crown.

The number of crowns placed on premolar teeth in the present study was small $(\mathrm{n}=2)$ and is too small a number from which to draw meaningful conclusions. However, one of the two crowns placed had failed, which may support the findings of Etemadi and Smales, ${ }^{12}$ in whose study there were substantially more failures in posterior teeth $(31.2 \%)$ than in anterior teeth $(7.8 \%)$ at five years. It may be considered that further work is indicated on the success of the dentine-bonded crown technique, employing ceramics of optimised physical properties which may still be etched in order to produce a micromechanically retentive fitting surface.

The retention of dentine-bonded crowns, obtained by means of the micromechanical retention of the lute to the restoration by etching the fitting surface of the crown with hydrofluoric acid in a manner similar to that in the porcelain veneer technique, ${ }^{18}$ treating the porcelain surface with a silane bond enhancing agent and the dentine surface with a dentine bonding agent, is apparently adequate. This luting procedure, which has been considered to produce a synergism between tooth and crown, ${ }^{3}$ may reduce the need for conventional means of retention, both in respect of the taper of the preparation, and also in respect of the height of the preparation. In this respect, the use of resin luting materials and in conjunction with adhesion to dentine has been considered, in a laboratory study, to make up for a lack of retention in teeth with excessive taper. ${ }^{19}$ The results of the present study, in which a high proportion of the crowns were placed on teeth with shortened clinical crowns as a result of tooth substance loss, would appear to indicate that the positive laboratory findings mentioned above can be translated into clinical practice.

The aesthetic characteristics of the crowns in the present study have been rated highly, possibly because there is no need for the technician to mask the grey metal core of a metal-ceramic restoration, or because of the improved light transmission when compared to metalceramic crowns or those luted with a non-translucent cement. ${ }^{6}$ The versatility of the dentine-bonded crown technique has previously been demonstrated in a series of case reports ${ }^{20}$ and is confirmed in the present study, in which a substantial proportion of the crowns were placed to restore teeth affected by tooth substance loss. Indeed, there seems little reason why all all-ceramic crowns are not luted with resin as described in this paper and as suggested by Christensen, ${ }^{21}$ although it is only those types of ceramic which may be etched to provide a micromechanically retentive fitting surface which receive the substantial advantages of reduced need for tooth preparation, the possibility of producing an excellent emergence profile and good aesthetics. The principal disadvantage has been the increased time required for placement, partly as a result of the need to etch the tooth and apply a dentine bonding agent, and to carry out occlusal adjustments and polishing after placement, rather than before, as with conventional crowns. However, the development of self-adhesive resin luting materials, such as RelyX Unicem (3M ESPE, Seefeld, Germany), could go some way to reducing the technique sensitivity of placing dentine-bonded crowns as these materials obviate the need for 


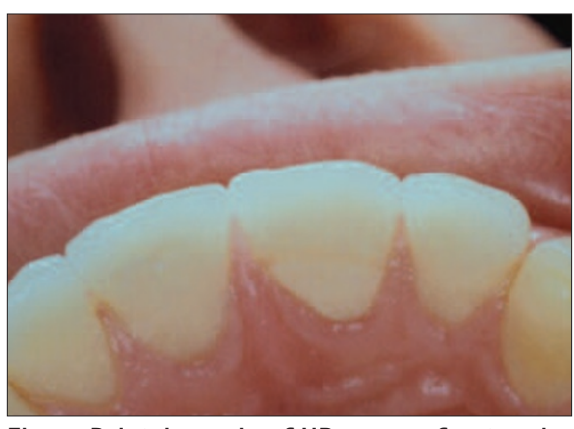

Fig. 5 Palatal margin of UR1 crown fractured at placement. Fragment pushed back into position, and replacement crown suggested to patient, who did not return for replacement crown. Fragment still in position, and tooth symptom free, at 3.5 years

separate etching and bonding steps. Initial clinical assessments of the performance of Rely-X Unicem are promising ${ }^{22,23}$ and a laboratory study has shown that dentine-bonded crowns luted with RelyX Unicem have similar fracture resistance to those luted with Mirage ABC/FLC, ${ }^{24}$ a material that has demonstrated good clinical performance. ${ }^{10}$

The incidence of pulp symptoms in the present study was nil, a similar finding to previous studies on dentine-bonded restorations, in which the need for endodontic treatment was reported as $2.4 \% 0^{10}$ and $0.7 \% .{ }^{25}$ This is in contrast to other publications which have traced the performance of crowns, ${ }^{26}$ with Saunders and Saunders, ${ }^{26}$ for example, finding a $19 \%$ incidence of periapical radiolucencies in crowned teeth which had not been root treated at the time of preparation. However, while Saunders and Saunders ${ }^{26}$ did not have any details of the status of the pulp in the teeth in their study at the time of crown placement, in contrast to the present study in which all teeth tested vital at the time of preparation, this would, nevertheless, appear to indicate that the dentine bonding technique used in the present study produces little or no challenge to the pulp, and, indeed, that the sealing of the dentinal tubules provides a beneficial effect and a lack of post-operative sensitivity.
Finally, one patient, who had received four crowns, returned for review at 3.5 years, having not attended for any previous recalls. At the fit appointment, a fragment of porcelain had detached from the gingival margin of one crown on its palatal surface during cementation: this had been placed back in position before the luting material had polymerised. This crown was not included in the study because of this occurrence. It is, however, of interest to note that this porcelain fragment, without any innate retention, had remained firmly in position (Fig. 5), demonstrating the effectiveness of the bond to etched ceramic via a dual-cure luting material and a dentine bonding agent, at least in this one instance.

\section{CONCLUSION}

The dentine bonded crowns assessed in this study showed excellent retention and low incidence of fracture at four years. This technique would appear to be suitable for a variety of clinical indications, including treatment of tooth substance loss, although the results presented in this study are relatively short term in relation to the anticipated life of restorations.

The author acknowledges the financial support of $3 M$ ESPE, St. Paul, MN, USA and wishes to thank the patients for their co-operation and $\mathrm{Mr}$ Alex Deacon at Glasgow Dental Hospital crown and bridge laboratory for his skill in constructing the crowns evaluated in this study.

1. Burke F J T. Fracture resistance of teeth restored with dentin-bonded crowns: the effect of increased tooth preparation. Quint Int 1996; 27: 115-121.

2. Burke F J T What's new in dentine bonding? Selfetch adhesives. Dent Update 2004; 31: 580-589.

3. Burke F J T, Watts D C. Fracture resistance of teeth restored with dentin-bonded crowns. Quint Int 1994; 25: 335-340.

4. Burke F J T. Maximising the fracture resistance of dentine-bonded all-ceramic crowns. J Dent 1999; 27: 169-173.

5. Crothers A J R, Wassell R W, Allen R. The resinbonded porcelain crown: a rationale for use on anterior teeth. Dent Update 1993; 20: 388-395.

6. Crocker W P. The cementation of porcelain jacket crowns with adhesive resins. Br Dent J 1992;
172: 64-67

7. Saunders W P, Patel S N, Burke F J T. Microleakage of dentin-bonded crowns placed with different luting materials. Am J Dent 1997; 10: 179-183.

8. Mirage technique booklet. Chameleon Dental Products, Kansas City, KN, USA.

9. Milosevic $A$, Jones $C$. Use of resin-bonded ceramic crowns in a bulimic patient with severe tooth erosion. Quint Int 1996; 27: 123-127.

10. Burke F J T, Qualtrough A J E, Wilson N H F. A retrospective evaluation of series of dentin-bonded ceramic crowns. Quint Int 1998; 29: 103-106.

11. Burke F J T, Qualtrough A J E. Follow-up retrospective evaluation of dentine bonded restorations. J Esthet Dent 2000; 12: 16-22.

12. Etemadi S, Smales R J. Survival of resin-bonded porcelain veneer crowns placed with and without metal reinforcement. J Dent 2005; 33: 139-145.

13. Bayindir $F, A$ kyil M S, Bayindir $Y Z$. Effect of eugenol and non-eugenol containing temporary cement on permanent cement retention and microhardness of cured composite resin. Dent Mater J 2003; 22: 592-599.

14. Peutzfelt $A$, Asmussen E. Influence of eugenolcontaining temporary cement on bonding of selfetching adhesives to dentin. J Adhes Dent 2006; 8: 31-34.

15. Ryge G. Clinical criteria. Int Dent J 1980; 30: 347-357.

16. van Dijken J W V, Hoglund-Aberg, Olofsson A-L. Fired ceramic inlays: a 6-year follow-up. J Dent 1998; 26: 219-225.

17. Fuzzi, Rappelli G. Ceramic inlays: clinical assessment and survival rate. J Adhes Dent 1999; 1: 71-79.

18. Soares C J, Soares P V, Pereira J C et al. Surface treatment protocols in the cementation of ceramic and laboratory-processed composite restorations: a literature review. J Esthet Restor Dent 2005; 17: 224-235.

19. Zidan O, Ferguson G C. The retention of complete crowns prepared with three different tapers and luted with four different cements. J Prosthet Dent 2003; 89: 565-571.

20. Burke F J T, Hussey D L, McCaughey D. Evaluation of the 1-year clinical performance of dentinbonded ceramic crowns and four case reports. Quint Int 2001; 32: 593-601.

21. Christensen $G J$ J. The rise of resin for cementing restorations. J Am Dent Assoc 1993; 124: 104-105.

22. Clinical Research Associates. Self-etching primer dual-cure resin cement. CRA Newsletter 2003; 27: 1-2.

23. Crisp R J, Burke F J T, Windmueller B. Handling evaluation of a self-adhesive universal resin cement by UK dental practitioners. AADR 2003; abstract 1279 .

24. Burke FJ T, Fleming G J, Windmueller B. Fracture strength of dentin-bonded crowns luted with a self-adhesive resin luting material. Eur J Prosthodont Rest Dent; in press.

25. van Dijken J W V, Hasselrot L., Ormin A et al. Restorations with extensive dentin/enamel bonded ceramic coverages. Eur J Oral Sci2001; 109: 222-229.

26. Saunders W P, Saunders E M. Prevalence of periradicular periodontitis associated with crowned teeth in an adult Scottish subpopulation. Br Dent J 1998; 185: 137-140. 